I0,000 $\AA$. In this case the dispersion was $150-200 \AA / \mathrm{mm}$. for $f / 2$. A. B. Severny (Ix) and G. S. Ivanov-Kholodny( 12 ) successfully photographed solar prominences and their infra-red spectra.

A. A. Kaliniak ( ( $\left._{3}\right)$ obtained infra-red photographs of the solar corona by means of a photo-electric image converter attached to the coronagraph of the Mountain Station of the Central Astronomical Observatory of the Academy of Sciences of the U.S.S.R. He also obtained similar photographs during the solar eclipse of 1954 .

At the Geophysical Institute of the Academy of Sciences of the U.S.S.R., B. A. Bagariatzky and M. I. Mordukhovitch (ro) have successfully photographed the spectra of aurorae up to II,000 $\AA$ by means of the prism spectrograph used earlier at the Crimean Astrophysical Observatory. Using an ordinary photo-electric image converter attached to an $f / 2$ diffraction spectrograph with a dispersion of $250 \AA / \mathrm{mm}$., B. A. Bagariatzky and N. I. Fedorova have obtained more detailed photographs of the infra-red spectra of aurorae and the night air-glow up to $I I, 000 \AA$. In the latter case the exposures reached 5-Io hr.

By means of a photo-electric image converter attached to an $f / \mathrm{I} \cdot 4$ camera of $90 \mathrm{~cm}$. focus, P. V. Scheglov (Sternberg Astronomical Institute) obtained at the Crimean Astrophysical Observatory a number of direct photographs of stars in the infra-red.

\title{
REFERENCES
}

(I) V. I. Krassovsky, C.R. Acad. Sci. U.R.S.S. 66, no. r, 1949.

(2) V. I. Krassovsky, C.R. Acad. Sci. U.R.S.S. 7o, no. 6, r950.

(3) V. I. Krassovsky, Publ. Crim. Obs. 5, roo, 1950.

(4) A. A. Kaliniak, V. I. Krassovsky, and V. B. Nikonov, C.R. Acad. Sci. U.R.S.S. 66, no. I, 1949.

(5) A. A. Kaliniak, V. I. Krassovsky, and V. B. Nikonov, Publ. Crim. Obs. 6, I 19, 1950.

(6) V. I. Krassovsky, C.R. Acad. Sci. U.R.S.S. 78, no. 3, I95I.

(7) V. T. Lukashenja and V. I. Krassovsky, C.R. Acad.Sci.U.R.S.S. 79, no. 2, I95I.

(8) V. I. Krassovsky and V. T. Lukashenja, C.R. Acad. Sci. U.R.S.S. 8o, no. 5, 195 I.

(9) V. T. Lukashenja and V. I. Krassovsky, C.R. Acad. Sci. U.R.S.S. 8I, no. 5, I 95 I.

(Io) B. A. Bagariatzky and M. I. Mordukhovitch, C.R. Acad. Sci. U.R.S.S. 82, no. I, I952.

(I I) A. B. Severny, C.R. Acad. Sci. U.R.S.S. 74, no. 2, 1950.

(I2) G. S. Ivanov-Kholodny, Publ. Crim. Obs. 8, I r5, I95I.

(13) A. A. Kaliniak, C.R. Acad. Sci. U.R.S.S. 72, 66r, 1950.

\section{COMPARISON OF IMAGE-TUBE SYSTEMS}

By A. D. CODE and R. H. HARDIE, Washburn Observatory and Lowell Observatory

The high quantum efficiency of the cathode in a photo-electric image device results in a greater number of statistically significant photo-events per unit time than can be obtained with an unaided photographic plate. The maximum information is contained in the initial photo-electron flux at the photocathode. The measurement or storage of these primary events represents the major problem in image-tube design.

A figure of merit or a detectability criterion for an image surface is derived on the basis of signal-to-noise ratio considerations. The performance of any specific image device is to be compared with this 'ideal' performance. The principal factors responsible for 'non-ideal' performance, namely, saturation and subsequent circuit noise, are examined. An expression for the sky-saturated case is derived which is equivalent to that presented by W. A. Baum in paper no. 3 .

It is suggested that the use of fine-grain plates, image magnification, or multiple exposures are practical methods of greatly extending the saturation limit. By these 
techniques, image converters such as those constructed by A. Lallemand should ultimately be capable of extending the limit of detection 2.5 magnitudes. On the other hand, devices which store the image electrically and utilize a scanning beam to read it are limited principally by the noise in the scanning beam rather than by saturation. If the scanning beam is to record images over a range of five magnitudes, the signal-to-noise ratio will be decreased at least by a factor of ten. This loss may be avoided by the introduction of sufficient multiplication (of the order of Ioo) in stages preceding the scanning process.

The choice between various methods should rest entirely upon which system most closely retains the initial signal-to-noise ratio of the photocathode for threshold objects. It appears that either the converter method reviewed by Lallemand in paper no. I or the electron-storage method reviewed by Morton in paper no. 2 might in fact be made to approach to within $\sqrt{ } 2$ of this signal-to-noise ratio. The advantages to be gained, however, are so great and the cost so small, in comparison with those achievable with larger telescope apertures, that it would seem foolish not to push development along several lines simultaneously.

\title{
8. DEVELOPMENT PROGRAMME ON PHOTO-ELECTRONIC IMAGE TUBES IN THE DEPARTMENT OF INSTRUMENT TECHNOLOGY, IMPERIAL COLLEGE OF.SCIENCE AND TECHNOLOGY, LONDON
}

\author{
By J. D. McGeE
}

Experimental work on a conventional Lallemand photographic image converter is being carried out in order to gain experience and to explore various possibilities.

An image converter is also being developed in which the electron image is formed on a fluorescent screen on the inner surface of a very thin (< IOO microns) glass window, I $\mathrm{mm} . \times 20 \mathrm{~mm}$. or smaller, capable of supporting atmospheric pressure. The photographic film is brought into contact with the outer surface of the thin glass. Thus, little fluorescent light is lost, and an efficiency comparable with that of the photographic image converter should be obtained. This is particularly designed for the registration of spectra.

Experimental work is in progress on a specialized television signal generating device. In the interest of developing a more efficient tube for astronomical purposes, we ignore the limitations and standardizations conventionally imposed on the design of commercial television tubes. In particular:

(I) The charging and discharging of the storage target can be sequential instead of simultaneous.

(2) The discharging can be done more slowly with the advantages of better signal-tonoise ratio and more efficient discharging of the integrated charges.

(3) The capacity of the electron-storage target can be increased, thereby enabling charge integration to be continued until the signals from very faint stars are large compared with the fluctuation (noise) in the background.

(4) The storage target can be made to have extremely good insulation, hence insuring no loss of image detail due to charge leakage over long periods.

The tube design suggested in March 1952 in the Journal of the Royal Society of Arts, no. 4869 , p. 343, and later at the Manchester Symposium on Astronomical Optics, April I955, is the basis of this work. Initially, a modified design is being tested in which a reversible storage target is mounted on pivots. After it is charged by photo-electrons incident from one direction, the target is then turned $180^{\circ}$ and the integrated charges are discharged by a conventional scanning beam incident from the opposite direction. The storage target obviously can be made double-sided, so that one surface is being charged while the other is being discharged. It may, for example, be a metal disk with a 